\title{
An Improved Control-Oriented Modeling of the Magnetic Field
}

\author{
Maxime Etiévant ${ }^{1}$, Aude Bolopion ${ }^{1}$, Stéphane Régnier ${ }^{2}$, Nicolas Andreff ${ }^{1}$
}

\begin{abstract}
This paper proposes a new control-oriented model to compute the magnetic field created by a coil. A major challenge for untethered microscale mobile robotics is the control of objects for precise and fast displacements. In this work, we propose to use an alternative implementation of a model based on elliptic integral functions to control magnetically actuated micro-robots. It allows to compute, quickly and accurately, the magnetic field even in the area close to the coil. This model is evaluated numerically and compared to classical approaches - dipole approximation, map-based interpolation and classical elliptic integral models - in terms of accuracy, computation time and memory requirement. Simulation results show that this works allows to have an accurate model in all the workspace by avoiding numerical issues encountered in previous works. It can be computed in a few milliseconds, making it the right candidate for closed-loop control of magnetically actuated micro-robots.
\end{abstract}

\section{INTRODUCTION}

Thanks to their size, untethered sub-millimeteric robots are expected to perform tasks inside hard-to-reach human body regions to do mini-invasive operations [1], cargo delivery [2] [3] [4] or micro-manipulation [5]. Magnetic fields are commonly used to wirelessly actuate and control these robots [6] [7]. Coils are good candidates to generate the magnetic fields [8] [9]. To strengthen the field effects, since the amplitude and direction of the field can be easily controlled by setting the appropriate current, one can increase the current circulating inside the coil. However, this solution presents the risk of overheating the coil and the necessity of cooling elements. Since the magnetic field quickly decreases as the distance to the coil increases, a second solution is to bring the coil as close as possible to the workspace. However, not all the classical magnetic models predict accurately the magnetic field close to the coil.

An accurate control of these magnetically actuated microrobots is often required, especially when they must perform delicate operations in the medical domain. A closed loop control is then implemented [10] [11]. These control loops are based on a model to calculate the magnetic force and torque applied on the robot by the magnetic field produced by the coil. A key step is thus to compute the magnetic field produced by the coil. Thanks to the exteroceptive feedback, closed-loop control can reject model errors to some extent. Therefore, the best control-oriented magnetic model is not

1 FEMTO-ST Institute, AS2M department, Univ. Bourgogne FrancheComté, Univ. de Franche-Comté/CNRS/ENSMM, 24 rue Savary, F-25000 Besançon, France. maxime . etievantefemto-st. fr

2 ISIR, INTERACTION, Univ. Pierre et Marie Curie, 4 Place Jussieu, 75005 Paris, France. necessarily the most accurate, but the one with the best tradeoff between speed and accuracy. Several models, based both on numerical and analytical approaches, have been proposed in the literature. Numerical models are based on maps of the magnetic field obtained either from finite element methods or from experimental measurement of the field. This first method allows a good accuracy at the cost of an important calculation time. Analytical methods are often based on dipole approximation, which offers a better computation time but constrain the spatial range of use [12], or on elliptical integrals [13], which can be complex to calculate. Hybrid approaches, such as in [14], can also be used: the authors use a map of the magnetic field obtained from finite element method and fit an analytical model based on the dipole approximation to these numerical values of the magnetic field. A similar approach is used in [15]. In this paper, we propose to use elliptic integral model proposed in [16] to be fast to compute and accurate in all the workspace, including the area close to the coil. It is thus evaluated and compared to classicals models and other existing elliptic integral models in term of computation time and accuracy.

This paper is organized as follows. Section II is devoted to the classical magnetic field modeling used in micro-robotics. Section III presents the elliptic integral model suited for robotic control. Models are then evaluated and compared in Section IV. Section V concludes the paper.

\section{MAGNeTIC FIELD MODELLING}

By modulating the magnetic field, it is possible to generate a force and a torque on a micro-robot gien by:

$$
\begin{aligned}
& \overrightarrow{f_{m}}=\int_{V_{m}} \nabla(\vec{m} \cdot \vec{B}) d V_{m}=\nabla(\vec{M} \cdot \vec{B}) \\
& \overrightarrow{\tau_{m}}=\int_{V_{m}}(\vec{m} \wedge \vec{B}) d V_{m}=\vec{M} \wedge \vec{B}
\end{aligned}
$$

where $V_{m}$ is the volume of the magnetic part of the robot under an applied magnetic field $\vec{B}, \vec{M}$ its magnetic moment and $\vec{m}$ the volumic density of magnetic moment, considered uniform in these equations.

The coils used to generate the magnetic field usually verify the following assumptions. The internal resistance of the coils being relatively small, the time constants associated with the circuit are marginal. That is why only the magneto-static domain is commonly studied. According to Maxwell's laws 


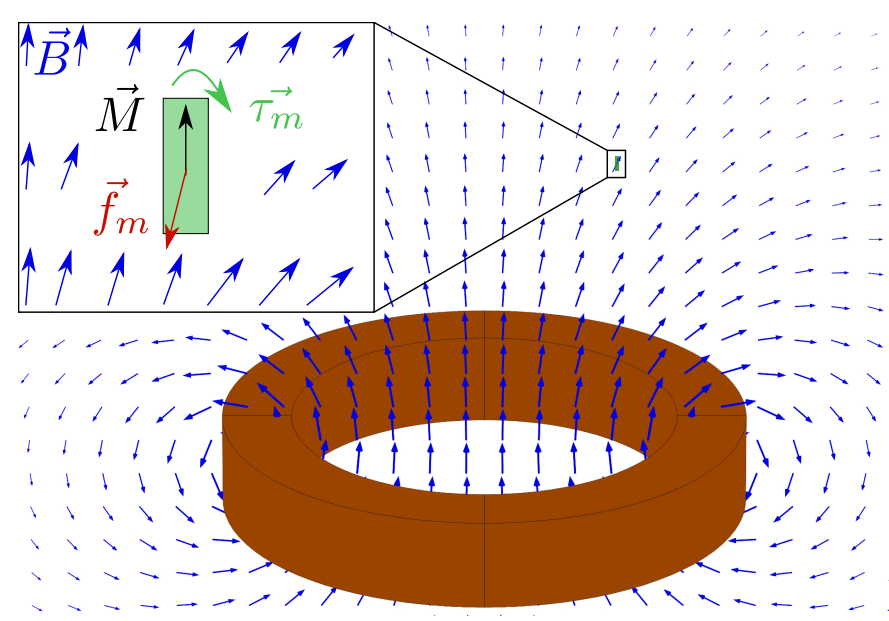

Fig. 1: Magnetic field generated by a coil and corresponding magnetic force and torque applied on a particle. The magnetic particle is represented by a green rectangle. Its magnetization is along its main axis. Under the effect of the magnetic field generated by the coil, the particle tends to align with the field lines (in blue) while it also experiences a magnetic force (red arrow).

and by splitting the magnetic field $\vec{B}$ into magnetization $\vec{M}$ and magnetic excitation $\vec{H}$ it comes:

$$
\left\{\begin{aligned}
\vec{\nabla} \wedge \vec{H} & =\vec{J} \\
\vec{\nabla} \cdot \vec{B} & =\overrightarrow{0} \\
\vec{B} & =\mu_{0}(\vec{H}+\vec{M})
\end{aligned}\right.
$$

where $\mu_{0}$ is the vacuum permeability and $\vec{J}$ the current density. In addition, it is considered that the magnetic field is linear with respect to the intensity circulating in the coil [14], often written as $\vec{B}=\overrightarrow{b_{i}} I$, where $I$ is the current circulating inside the coil and $b_{i}$ the unit-current field.

To compute the magnetic field produced by several coils, it is often considered that the superposition principle holds. The magnetic field produced by $n$ coils is then the sum of the magnetic fields $\overrightarrow{B_{n}}$ produced by each coil. This is true when the coils do not have core, or if they have soft magnetic cores which magnetization stay in the linear domain and if the hysteresis is neglected as in [17]. The total field is then:

$$
\vec{B}=\sum \vec{B}_{n}=\sum \overrightarrow{b_{i n}} I_{n}
$$

In the following, we will thus concentrate on models able to compute the magnetic field produced by a single coreless coil powered by a current of $1 \mathrm{~A}$. In addition, assuming that the coil is made of circular loops, the problem is axisymetric.

\section{A. Numerical method}

A first method consists in creating a unit-current field map for each coil. The magnetic field generated by several coils is computed thanks to simple mathematical operations (symmetry, translation, rotation and scaling by the current) before superposition on each $\overrightarrow{b_{i}}$.

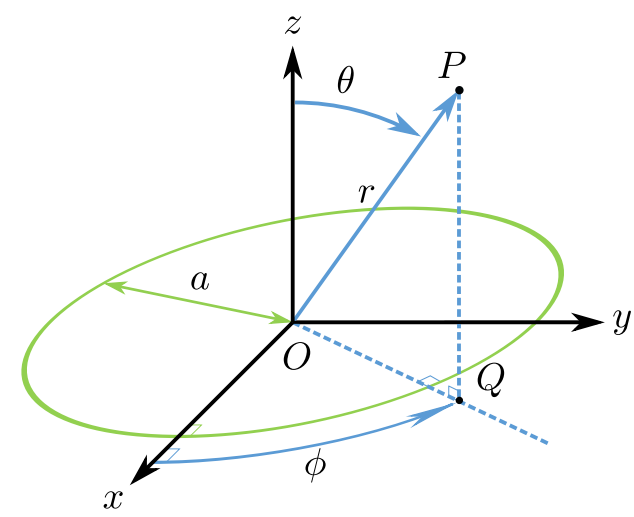

Fig. 2: Current loop parameters

To obtain these unit-current field maps, one can either experimentally measure the magnetic field using a hall effect sensor or use finite-element software to compute the map. The resulting map being discretized in a grid of points, it is then necessary to use interpolation to evaluate the field in an arbitrary position.

\section{B. Dipole model}

The solutions above being numerical, one can nevertheless turns to an analytical approach to solve this problem. The most often used is the dipolar approximation. The dipole model is based on the Biot-Savart law (6) and consists in a simplification of the problem by considering the coil (or each turn) as a punctual magnetic dipole. Consider a circular current loop as in [18] parametrized using the spherical coordinate system as in Fig. 2 with:

$$
\left\{\begin{array}{lll}
r=\sqrt{x_{p}^{2}+y_{p}^{2}+z_{p}^{2}} ; & & r \geq 0 \\
\phi=\arctan \left(y_{p}, x_{p}\right) ; & & \phi \in[0 ; 2 \pi[ \\
\theta=\arccos \left(z_{p} / r\right) ; & & \theta \in[0 ; \pi]
\end{array}\right.
$$

In this figure, the magnetic field created in point $P=$ $\left(x_{p}, y_{p}, z_{p}\right)$ by a circular loop of radius $a$ centered in $O$ is considered. The magnetic field provided by this loop is given by:

$$
\overrightarrow{B(P)}=\frac{\mu_{0} I}{4 \pi} \oint_{Q} \frac{I \overrightarrow{d l} \wedge(\overrightarrow{Q P})}{\|\overrightarrow{Q P}\|^{3}}
$$

This expression is true for points $P$ situated far away from the coil, (located at a distance much greater than the coil radius $r \gg a$ ). After simplification, the magnetic field is expressed in spherical coordinates as follows:

$$
\left\{\begin{aligned}
B_{r}(r, \theta) & =\frac{\mu_{0} I a^{2} \cos (\theta)}{2 r^{3}} \\
B_{\theta}(r, \theta) & =\frac{\mu_{0} I a^{2} \sin (\theta)}{4 r^{3}} \\
B_{\phi} & =0
\end{aligned}\right.
$$




\section{Elliptic integral}

1) Direct expression of $\vec{B}$ : The magnetic field given by the Biot-Savart law (Eq. (6)) can also be solved using incomplete elliptic integrals as in [13]. Using the parameters presented in Fig. 2 the magnetic field in cartesian coordinates is given by:

$$
\begin{aligned}
B_{x}= & \frac{\mu_{0} I}{2 \pi} \frac{1}{\sqrt{a^{2}+r^{2}+2 a r \sin (\theta)}}\left[\mathcal{F}\left(\frac{\pi}{2}, k^{2}\right)\right. \\
& \left.+\frac{a^{2}-r^{2}}{a^{2}+r^{2}-2 a r \sin (\theta)} \mathcal{E}\left(\frac{\pi}{2}, k^{2}\right)\right] \\
B_{z}= & \frac{\mu_{0} I}{4 \pi} \frac{1}{\tan (\theta) \sqrt{a^{2}+r^{2}-2 a r \sin (\theta)}} \\
& {\left[\frac{a^{2}+r^{2}}{\left(a^{2}+r^{2}+2 a r \sin (\theta)\right)}\left(\mathcal{E}\left(\frac{\pi}{4}, k^{2}\right)+\mathcal{E}\left(\frac{3 \pi}{4}, k^{2}\right)\right)\right.} \\
& \left.-\left(\mathcal{F}\left(\frac{\pi}{4}, n^{2}\right)+\mathcal{F}\left(\frac{3 \pi}{4}, n^{2}\right)\right)\right]
\end{aligned}
$$

where $\mathcal{E}$ and $\mathcal{F}$ are the incomplete elliptic integral functions of first and second kind [19]:

$$
\begin{array}{ll}
\mathcal{F}(\phi, k)=\int_{0}^{\sin \phi} \frac{1}{\sqrt{\left(1-t^{2}\right)\left(1-k^{2} t^{2}\right)}} d t & =\int_{0}^{\phi} \frac{1}{\sqrt{\left(1-k^{2} \sin ^{2} \theta\right)}} d \theta \\
\mathcal{E}(\phi, k)=\int_{0}^{\sin \phi} \frac{\sqrt{\left(1-k^{2} t^{2}\right)}}{\sqrt{\left(1-t^{2}\right)}} d t & =\int_{0}^{\phi} \sqrt{\left(1-k^{2} \sin ^{2} \theta\right)} d \theta
\end{array}
$$

$k$ and $n$ are defined in [13] by:

$$
\begin{aligned}
& k^{2}=\frac{4 a r \sin (\theta)}{a^{2}+r^{2}+2 a r \sin (\theta)} \\
& n^{2}=\frac{-4 a r \sin (\theta)}{a^{2}+r^{2}-2 a r \sin (\theta)}
\end{aligned}
$$

2) Formulation using the magnetic potential vector: To avoid the use of incomplete elliptic integral functions as in Sec. II-C1 and then reduce the computing time, it is possible to resort only to complete elliptic integral functions, which are faster to compute since the integration bounds are fixed. According to Maxwell's equation, the divergence of the magnetic field is identically null, so it can be assimilated to the curl of a vector field called magnetic potential vector $\vec{A}$. Indeed, as the magnetic field is orthogonal to the $\vec{\nabla}$ operator, it can be written as the cross product between $\vec{\nabla}$ operator and a vector (here the magnetic potential vector).

$$
\begin{aligned}
\vec{\nabla} \cdot \vec{B} & =\overrightarrow{0} \\
\vec{\nabla} \wedge \vec{A} & =\vec{B}
\end{aligned}
$$

Knowing the $A_{\phi}$ expression, one obtains the magnetic field $\vec{B}=\left(B_{r}, B_{\theta}, B_{\phi}\right)^{T}$ thanks to the expression of the curl operator in spherical coordinates:

$$
\left\{\begin{aligned}
B_{r}(r, \phi) & =\frac{1}{r \sin (\theta)} \frac{\partial}{\partial \theta}\left(\sin \theta A_{\phi}\right) \\
B_{\theta}(r, \phi) & =\frac{-1}{r} \frac{\partial}{\partial r}\left(r A_{\phi}\right) \\
B_{\phi} & =0
\end{aligned}\right.
$$

In [18], Jackson shows that the expression of the magnetic potential vector created by a circular loop expressed in any point is given in spherical coordinates by:

$$
\begin{aligned}
A_{r} & =A_{\theta}=0 \\
A_{\phi}(r, \phi) & =\frac{\mu_{0} I}{\pi} \frac{a}{\sqrt{a^{2}+r^{2}+2 a r \sin (\theta)}}\left[\frac{\left(2-k^{2}\right) K(k)-2 E(k)}{k^{2}}\right]
\end{aligned}
$$

where $K$ and $E$ are respectively complete elliptic integral of the first and second kind defined as follows:

$$
K(k)=\mathcal{F}\left(\frac{\pi}{2}, k\right) ; \quad E(k)=\mathcal{E}\left(\frac{\pi}{2}, k\right) .
$$

Equation (12) allows to know the magnetic field at any point in the space, but one of the reasons that explains its rare use is certainly its sensitivity to digital errors as noticed in [16]. Close to the coil or along the symmetry axis (i.e. $k \ll 1$ ), $A_{\phi}$ is badly computed which leads to a loss of accuracy. As stated above, these regions are however of great interest for robotic manipulation as they correspond to the center of the workspace or to areas of high magnitude forces and torques. To overcome this issue, Schill proposes continuity extension when $k \ll 1[16]$.

To compute the magnetic field given in Eq. (12) the magnetic potential vector $\left(A_{\phi}\right.$ component) must be differentiated. To do so, Schill proposes in [16] to use elliptical integrals of first and second but also third kind [19]. This solution is recalled below.

\section{PROPOSED IMPROVED ELLIPTIC INTEGRAL FORMULATION}

As noticed by Schill [16], the classical elliptic integral models remain sensitive to numerical errors. To compute the magnetic field given in Eq. (12) from the magnetic potential vector (Eq. (13)) we propose here a method only based on complete elliptic integral functions of the first and second kind with a continuity extension for $k \ll 1$ (when $r \ll 1$, $\sin (\theta) \ll 1$ or $r \gg a$ ). Notice that the derivation below is slightly different from [16], as no third kind elliptic integral function is needed, which yields different performances. This will be shown in the next section.

As noted previously, to compute the magnetic field it is necessary to differentiate the magnetic potential vector $\left(A_{\phi}\right.$ component). To do so, the derivative of the $E(k)$ and $K(k)$ function with respect to $k$ must be computed.

It is possible to demonstrate that:

$$
\left\{\begin{array}{l}
\frac{\partial E}{\partial k}(k)=\frac{E(k)-K(k)}{k} \\
\frac{\partial K}{\partial k}(k)=\frac{E(k)}{k\left(1-k^{2}\right)}-\frac{K(k)}{k}
\end{array}\right.
$$

The magnetic field given in spherical coordinates (Eq. (12)) can be computed from the magnetic potential vector (Eq. (13)) 
using the derivative of the elliptic integral functions (Eq. (15)):

$$
\begin{aligned}
B_{r}(r, \theta) & =\frac{\mu_{0} I}{\pi} \frac{a^{2}}{\sqrt{a^{2}+r^{2}+2 a r \sin (\theta)}} \frac{E(k) \cos \theta}{a^{2}+r^{2}-2 r a \sin \theta} \\
B_{\theta}(r, \theta) & =\frac{\mu_{0} I}{\pi} \frac{1}{\sqrt{a^{2}+r^{2}+2 a r \sin (\theta)}} f(r, \theta) \\
B_{\phi} & =0 \\
f(r, \theta) & =\left[\frac{E(k)\left(r^{2}+a^{2} \cos (2 \theta)\right)}{\left(a^{2}+r^{2}-2 r a \sin \theta\right) 2 \sin \theta}-\frac{K(k)}{2 \sin \theta}\right]
\end{aligned}
$$

We thus come up with an analytical expression which uses only complete elliptic integral functions of first and second kind.

Remark: These expressions are not defined for $k=0$. However, when $k \ll 1$, the continuity extension is done by using:

$$
\begin{aligned}
& K(k)=\frac{\pi}{2}+k^{2} \frac{\pi}{8}+k^{4} \frac{9 \pi}{128}+o\left(k^{4}\right) \\
& E(k)=\frac{\pi}{2}-k^{2} \frac{\pi}{8}-k^{4} \frac{3 \pi}{128}+o\left(k^{4}\right)
\end{aligned}
$$

which leads to:

$$
\frac{\left(2-k^{2}\right) K(k)-2 E(k)}{k^{2}}=k^{2} \frac{\pi}{16}+o\left(k^{4}\right)
$$

With this result, the magnetic potential vector $A_{\phi}$ (Eq.(13)) is simplified and the magnetic field $\vec{B}$ (Eq. (12)) becomes:

$$
\left\{\begin{aligned}
B_{r}(r, \theta) & =\frac{\mu_{0} I a^{2}}{4} \cos (\theta) \frac{\left(2 a^{2}+\sin (\theta) a r+2 r^{2}\right)}{\left(a^{2}+2 \sin (\theta) a r+r^{2}\right)^{5 / 2}} \\
B_{\theta}(r, \theta) & =-\frac{\mu_{0} I a^{2}}{4} \sin (\theta) \frac{\left(2 a^{2}+\sin (\theta) a r-r^{2}\right)}{\left(a^{2}+2 \sin (\theta) a r+r^{2}\right)^{5 / 2}} \\
B_{\phi} & =0
\end{aligned}\right.
$$

Remark: When $r \gg a$, this expression is simplified into the dipole approximation (7).

\section{COMPARISON OF THE MODELS}

In this section, the models presented above are evaluated in terms of accuracy, computation time and memory requirements.

\section{A. Simulation conditions}

To evaluate and compare the models, the parameters of the simulations will be based on a classical micro-robotic system. We consider a magnetic particle actuated by several coreless coils. As mentionned before, the challenge to control this particle is to compute quickly and accurately the magnetic field produced by the coils at the position of the particle. As explained in the previous section, this problem can be reduced to the computation of the magnetic field produced by a single coil for a unitary current. As the problem is axi-symetric, it is sufficient to perform the analysis on a plane containing the axis of the coil and the particle. A sketch of the simulated scene is given in Fig 2. The coil is centered in $[x, y, z]=[0,0,0]$ with the main axis along the $\mathrm{z}$-axis. Its dimensions are given

\begin{tabular}{lcc}
\hline Parameter & Value & Unit \\
\hline Coil mean diameter & 0.1 & $\mathrm{~m}$ \\
Number of loops & $500(25$ axially, 20 radially $)$ & - \\
Wire diameter & 0.8 & $\mathrm{~mm}$ \\
Current & 1 & $\mathrm{~A}$ \\
Coil center position & {$[0 ; 0 ; 0]$} & {$[\mathrm{m} \mathrm{m} \mathrm{m}]$} \\
\hline
\end{tabular}

TABLE I: Parameters of the coil used in the simulations.

in Table I. The workspace is a $20 \mathrm{~cm}$-side square centered on the coil. The models have been implemented on a computer equipped with an Intel Core i5-7600 processor at $3.5 \mathrm{GHz}$ and 16 Go of RAM. The Matlab R2016b software has been used.

As mentioned in Sec. II-A, the mapping methods necessitate to first get the values of the magnetic field at given points of a mesh. In this paper these numerical values are obtained from a finite element simulation performed on the COMSOL MultiPhysics software. A map of the magnetic field generated by a coil powered with a unitary current is obtained. To get the magnetic field at any arbitrary position, an interpolation is made. This computation step is time consuming, and a balance must be found between fineness of the mesh (to reduce the interpolation time) and the memory used by the computer (and therefore the time related to the search for points of interest). Here, a resolution $10 \mu \mathrm{m}$ was chosen, corresponding to $1 \%$ of the length of a millimetric robot. The interpolation is performed by the MATLAB interp 2 function which performs a 2-dimensional interpolation. For the analytical methods, each loop is considered independently and the total magnetic field is obtained using the superposition principle.

For each of the three methods, the time needed to evaluate the magnetic field at a given point (randomly selected in the workspace) is measured. To have reliable statistics, all the operation is repeated 180 times. In addition, the memory used and the model accuracy in term of orientation and intensity are confronted.

To be comparable, the different algorithms were fairly developed but migth still be optimized. In particular, the computation time needed for the mapping method seems to be important and should be reduced by replacing interp 2 function by a tailored method.

\section{B. Results}

The comparison between the different models in terms of computation time and memory requirements is given in Table II. Since all the numerical values of the magnetic field must be stored, the mapping approach requires more storage capacity. It is also long to compute: one can note a ratio of 95 between the computation time of the mapping model and that of the dipole model. This is propably due mainly to the interpolation that must be performed to compute the magnetic field at a given point.

All the numerical approaches present a similar memory requirement, limited to a few kilooctet. The direct computation of the magnetic field, as presented in [13], suffers from a huge computing time. This is mainly due to the use of incomplete elliptic integral. By using complete elliptic integral functions 


\begin{tabular}{lccc}
\hline & $\begin{array}{c}\text { Computation time } \\
(\mathrm{ms} / \text { point })\end{array}$ & $\begin{array}{c}\text { Standard deviation } \\
(\mathrm{ms})\end{array}$ & $\begin{array}{c}\text { Memory used } \\
(\mathrm{ko})\end{array}$ \\
\hline Mapping & 162 & 1.583 & $\geq 1400$ \\
Dipole & 1.7 & 0.163 & $\leq 6$ \\
Wong's formulation & 1164.5 & 7.1 & $\leq 7$ \\
Schill's formulation & 241.4 & 2.5 & $\leq 7$ \\
Extended formulation & 3.6 & 0.147 & $\leq 7$ \\
\hline
\end{tabular}

TABLE II: Computational comparison of different models.

of first, second and third kind as presented in [16], the calculation time is divided by a factor of about 5 but remains greater than a few hundred milliseconds. The computation time of the dipole model and of the proposed model are limited to a few milliseconds. They are thus both compatible real time computation of the magnetic field, and thus with closed-loop control.

To evaluate the accuracy of each model the angular deviation and norm error of the computed magnetic field are studied. All the models that involve elliptic integrals to compute the magnetic field (direct expression of $B$, formulation using the magnetic potential vector and the proposed approach) show very similar results in terms of accuracy, with relative difference between these three models lower than $10^{-10 \%}$. Since this is not significant and to facilitate the reading they will thus be considered jointly for the evaluation of accuracy. To evaluate the magnetic field computed with the different models, a reference is necessary. As in most articles in the robotics community, the result of the finite element method will be considered as a reference [14] [20].

Figures 3 and 4 presents the comparison of the dipole model and the models involving elliptic integrals compared to finite elements in a $x z$ plane including the coil axis. Fig. 3 gives the relative difference in terms of norm between the results obtained by finite element and the dipole approximation (resp the elliptic integral method) in the domain close to the coil. Fig. 4, the angle formed between these two results is compared. To compare the different models in term of the magnitude of the computed magnetic field, isovalues of the norm deviation between a given model and the reference (values obtained from finite element simulation). As expected, the deviation between the dipole model and the finite elements is extremely important near the axis of the coil or for positions close to the coil. For example, for the point located on the axis of the coil at a distance equal to the diameter of the coil, the dipole model presents an error of $1700 \%$ compared to finite elements. This is due to the fact that the hypothesis under which the dipole model is valid are not more validated. In comparison, the models involving elliptic integrals (direct expression of $B$, formulation using the magnetic potential vector and the proposed approach) have a relative difference of less than 5\% compared to the finite element model in the whole workspace. This latent error can notably be explained by numerical residuals.

Another important point to evaluate the accuracy is the error between the orientation of the computed magnetic field and the one obtained from finite element simulation. Indeed, an angular deviation induces an error on both the computed force and torque applied to the robot. The bottom subfigures (Fig. 3) compare the models. Isovalues corresponding to 5, 10, 20, 30, 45 and $90^{\circ}$ are represented. For the dipole model, the angular error increases as the distance with the coil decreases. Errors can reach $90^{\circ}$, which means that the computed magnetic field is perpendicular to the magnetic field obtained from finite element simulation. Again, this is due to the fact the the hypothesis under which the dipole model is true are no more valid. The angular error between the elliptic integral model and finite element simulations is represented in bottom-right Figure. It is less than $5^{\circ}$ in most of the workspace, and less than $20^{\circ}$ in all the workspace. The fact that errors increase with the distance to the coil might be partly explained by numerical residues.

As a conclusion, mapping-based models are costly to implement and only reveal their potential when the geometry of the coil is complex or when cores are used. When the magnetic field is computed far from the coil, a simplified model based

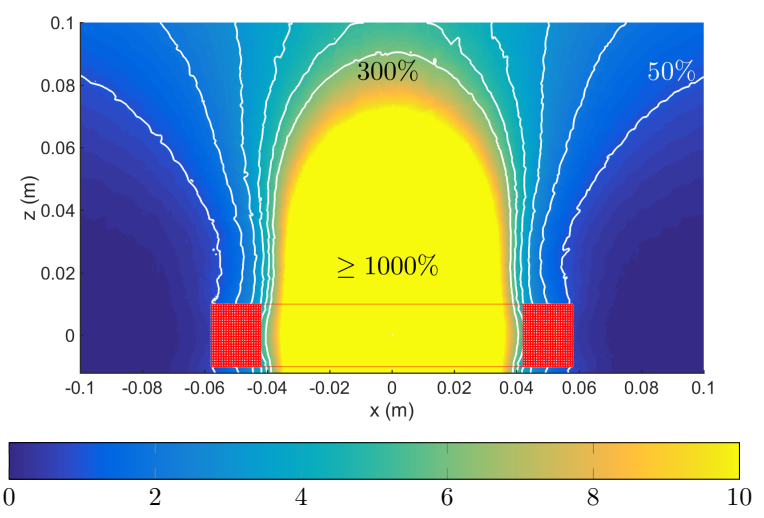

(a) Dipole model

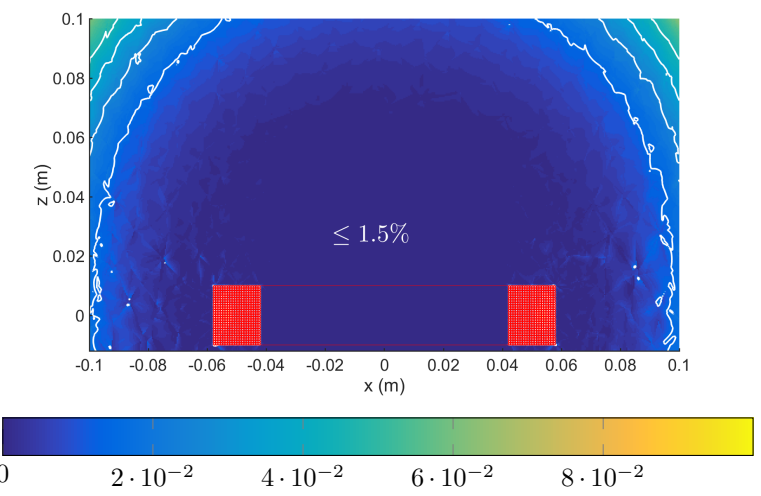

(b) Elliptic integral model

Fig. 3: Relative errors between magnitude of the computed magnetic field w.r.t the result of a finite element simulation (unit-less). Top: Dipole field computed w.r.t finite element result. Bottom: Elliptic integrals method result w.r.t finite element result. 


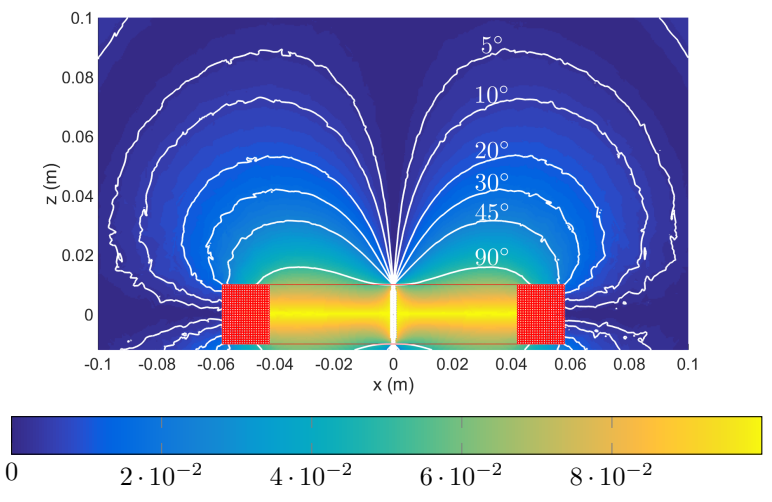

(a) Dipole model

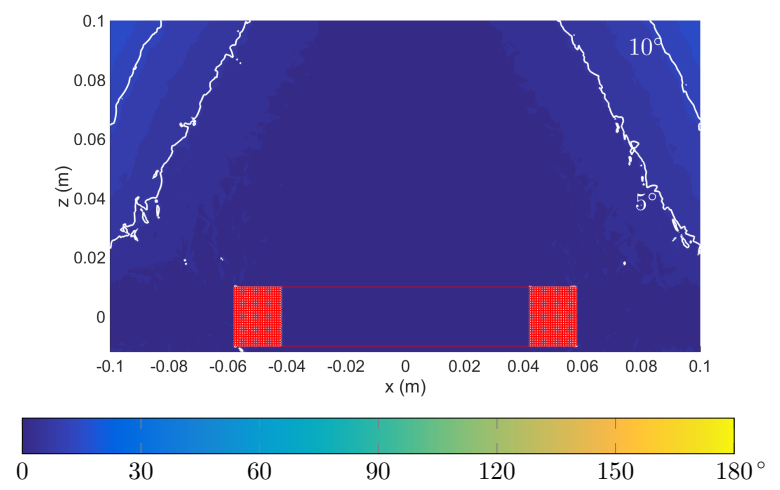

(b) Elliptic integral model

Fig. 4: Angular errors between the computed magnetic field w.r.t the result of a finite element simulation $\left(^{\circ}\right)$. Top: Dipole result w.r.t finite element result. Bottom: Elliptic integrals method result w.r.t finite element result.

on the dipole approximation allows a significant computing gain and a good accuracy. Models involving elliptic integral functions are accurate in all the workspace, including areas close to the coil. However, the models in the litterature (based on the direct evaluation of $B$ or using the magnetic potential vector) remains as slow as mapping-based models. In comparison, the proposed approach presents a similar accuracy, but the computing time is at least 65 times less. Based on the computed magnetic field, the force and torque applied on magnetically actuated robots can be computed using Eq. (16) in Eq. (1) and (2).

Our model is thus a good compromise between accuracy and computation time, making it the right candidate for closed-loop control of magnetically actuated micro-robots.

\section{CONCLUSION AND FUTURE WORK}

In this paper, we proposed a new model to compute accurately the magnetic field in all the workspace, including in the area close to the coil. It is based on the use of elliptic integral extended by continuity to compute the magnetic field based on the expression of the magnetic potential vector. Compared to existing approaches involving elliptic integral, only complete elliptic integrals, and restrained to the first and second kinds are used. This allows computing times at least 65 times faster than existing elliptic integral-based methods. It can thus be computed on a few milliseconds only making it suitable real-time control. Contrary to the classical dipole model, it is accurate in all the workspace, including close to the coil. This is thus the only model compatible with closed-loop control of magnetically actuated robot that provides accurate results in all the workspace.

In future work, we will investigate the influence of a soft core in the electromagnet.

\section{ACKNOWLEDGMENT}

This project was supported by the EUR EIPHI program (Contract No. ANR-17-EURE-0002), by the COErCIVe région Bourgogne Franche-Comté project and by the French Agence Nationale de la Recherche through the Multiflag project (contract "ANR-16-CE33-0019").

\section{REFERENCES}

[1] B. J. Nelson, I. K. Kaliakatsos, and J. J. Abbott, "Microrobots for Minimally Invasive Medicine," Annual Review of Biomedical Engineering, vol. 12, no. 1, pp. 55-85, 2010. [Online]. Available: https://doi.org/10.1146/annurev-bioeng-010510-103409

[2] F. Qiu, S. Fujita, R. Mhanna, L. Zhang, B. R. Simona, and B. J. Nelson, "Magnetic Helical Microswimmers Functionalized with Lipoplexes for Targeted Gene Delivery," Advanced Functional Materials, vol. 25, no. 11, pp. 1666-1671, Jan. 2015. [Online]. Available: https://onlinelibrary.wiley.com/doi/abs/10.1002/adfm.201403891

[3] I. S. Khalil, V. Magdanz, S. Sanchez, O. G. Schmidt, and S. Misra, "Biocompatible, accurate, and fully autonomous: a sperm-driven microbio-robot," Journal of Micro-Bio Robotics, vol. 9, no. 3-4, pp. 79-86, 2014.

[4] M. Medina-Sánchez, L. Schwarz, A. K. Meyer, F. Hebenstreit, and O. G. Schmidt, "Cellular Cargo Delivery: Toward Assisted Fertilization by Sperm-Carrying Micromotors," Nano Letters, vol. 16, no. 1, pp. 555-561, Jan. 2016. [Online]. Available: http://dx.doi.org/10.1021/acs.nanolett.5b04221

[5] M. Sitti, H. Ceylan, W. Hu, J. Giltinan, M. Turan, S. Yim, and E. Diller, "Biomedical Applications of Untethered Mobile Milli/Microrobots," Proceedings of the IEEE, vol. 103, no. 2, pp. 205-224, Feb. 2015.

[6] S. Chowdhury, W. Jing, and D. J. Cappelleri, "Controlling multiple microrobots: recent progress and future challenges," Journal of MicroBio Robotics, vol. 10, no. 1-4, pp. 1-11, 2015.

[7] T. Xu, C.-I. Vong, B. Wang, L. Liu, X. Wu, and L. Zhang, "Rotating soft-tail millimeter-scaled swimmers with superhydrophilic or superhydrophobic surfaces," in Biomedical Robotics and Biomechatronics (BioRob), 2016 6th IEEE International Conference on. IEEE, 2016, pp. 502-507.

[8] J. J. Abbott, K. E. Peyer, M. C. Lagomarsino, L. Zhang, L. Dong, I. K. Kaliakatsos, and B. J. Nelson, "How Should Microrobots Swim?" The International Journal of Robotics Research, vol. 28, no. 11-12, pp. 1434-1447, Nov. 2009. [Online]. Available: https://doi.org/10.1177/0278364909341658

[9] S. Tottori, L. Zhang, F. Qiu, K. K. Krawczyk, A. Franco-Obregón, and B. J. Nelson, "Magnetic Helical Micromachines: Fabrication, Controlled Swimming, and Cargo Transport," Advanced Materials, vol. 24, no. 6, pp. 811-816, Feb. 2012. [Online]. Available: http://onlinelibrary.wiley.com/doi/10.1002/adma.201103818/abstract

[10] S. Martel, M. Mohammadi, O. Felfoul, Z. Lu, and P. Pouponneau, "Flagellated magnetotactic bacteria as controlled mri-trackable propulsion and steering systems for medical nanorobots operating in the human microvasculature," The International journal of robotics research, vol. 28, no. 4, pp. 571-582, 2009.

[11] A. Oulmas, N. Andreff, and S. Régnier, "Closed-loop 3d path following of scaled-up helical microswimmers," in 2016 IEEE International Conference on Robotics and Automation (ICRA), May 2016, pp. 1725-1730. 
[12] P. Ryan and E. Diller, "Five-degree-of-freedom magnetic control of micro-robots using rotating permanent magnets," in Robotics and $\mathrm{Au}$ tomation (ICRA), 2016 IEEE International Conference on. IEEE, 2016, pp. $1731-1736$.

[13] D. Wong, E. B. Steager, and V. Kumar, "Independent Control of Identical Magnetic Robots in a Plane," IEEE Robotics and Automation Letters, vol. 1, no. 1, pp. 554-561, Jan. 2016.

[14] M. P. Kummer, J. J. Abbott, B. E. Kratochvil, R. Borer, A. Sengul, and B. J. Nelson, "OctoMag: An Electromagnetic System for 5-DOF Wireless Micromanipulation," IEEE Transactions on Robotics, vol. 26, no. 6, pp. 1006-1017, Dec. 2010.

[15] E. Diller, J. Giltinan, and M. Sitti, "Independent control of multiple magnetic microrobots in three dimensionsThe International Journal of Robotics Research," 2013. [Online]. Available: http://journals.sagepub.com/doi/abs/10.1177/0278364913483183

[16] R. A. Schill, "General relation for the vector magnetic field of a circular current loop: a closer look," IEEE Transactions on Magnetics, vol. 39, no. 2, pp. 961-967, Mar. 2003.

[17] A. Denasi and S. Misra, "Independent and Leader \#x2013;Follower Control for Two Magnetic Micro-Agents," IEEE Robotics and Automation Letters, vol. 3, no. 1, pp. 218-225, Jan. 2018.

[18] J. D. Jackson, Classical Electrodynamics, 3rd ed. New York, $\{\mathrm{NY}\}$ : Wiley, 1999.

[19] M. Abramowitz and I. A. Stegun, Handbook of mathematical functions: with formulas, graphs, and mathematical tables. Courier Corporation, 1965, vol. 55.

[20] J. Li, E. S. Barjuei, G. Ciuti, Y. Hao, P. Zhang, Q. Shi, A. Menciassi, Q. Huang, and P. Dario, "Analytical magnetic model applied to endoscopic robots design: A ready-to-use implementation and a case of study," in Information and Automation (ICIA), 2016 IEEE International Conference on. IEEE, 2016, pp. 1618-1623. 